\title{
Erratum to: Co-spray Drying with HPMC as a Platform to Improve Direct Compaction Properties of Various Tablet Fillers
}

\author{
JinZhi Li, ${ }^{1,2}$ LiJie Zhao, ${ }^{3}$ Xiao Lin,,${ }^{1,4}$ Lan Shen, ${ }^{1}$ and Yi Feng ${ }^{3,4}$
}

\section{Erratum to: AAPS PharmSciTech}

\section{DOI 10.1208/s12249-017-0794-1}

The publisher regrets not revising the caption for Fig. 1 as instructed by the author at the proof stage of the article. The revised caption for Fig. 1 appears below. The original article was corrected.

Fig. 1. Three dimensional response surface plots of the developed models. a starch-HPMC powders. b calcium hydrogen phosphate dihydrate-HPMC powders. c mannitol-HPMC powders. \% $A$ HPMC concentration in products $(w / w) ; \% B$ concentration of solid content in feeds $(w / v) ; \rho b$ bulk density; $\rho t a$ tap density; $D(0.5)$ the median particle size; TS tensile strength; $D T P$ disintegration time of tablets composed of $96.5 \%$ the coprocessed excipient and 3.5\% PVPP; DT disintegration time of tablets containing the co-processed excipient only; $P y$ yield pressure; $E s p$, unit effective compaction work.

The online version of the original article can be found at http:// dx.doi.org/10.1208/s12249-017-0794-1

\footnotetext{
${ }^{1}$ College of Chinese Materia Medica, Shanghai University of Traditional Chinese Medicine, Shanghai, 201203, People's Republic of China.

${ }^{2}$ College of Chinese Materia Medica, Zhejiang Pharmaceutical College, Ningbo, 315100, People's Republic of China.

${ }^{3}$ Engineering Research Center of Modern Preparation Technology of TCM of Ministry of Education, Shanghai University of Traditional Chinese Medicine, Shanghai, 201203, People's Republic of China.

${ }^{4}$ To whom correspondence should be addressed. (e-mail: duotang@163.comyy090503@163.com)
} 